\title{
Microscopic Photometry and Its Parameterization for Objective Evaluation of Aesthetics of Woven Fabrics
}

\begin{abstract}
One of the important end-use performance parameters for fabrics and other textile products are the fabric hands and fabric aesthetics. Our previous research attempted to elucidate the factors that affect the fabric aesthetics using factor analysis and identified two principal factors tentatively labelled as 'lustre and depth sensation' and 'surface roughness sensation'. The focus of the second step in the study of fabric aesthetics is on the investigation of the objective trends in the performance evaluation of textiles. In this study, we developed our own experimental equipment based on microscopic goniophotometric principles and its parameterization method. Subsequently, we attempted to define an index based on the distribution of the reflection intensity of woven fabrics and its characterization in order to evaluate the principal factors governing fabric aesthetics. On the basis of the results, we infer that microscopic goniophotometry and its parameterization is a more viable approach than conventional macroscopic goniophotometry for the quantitative evaluation of the principal factors governing fabric aesthetics.
\end{abstract}

Key words: Fabric aesthetics, Quantitative evaluation, Microscopic goniophotometry, Principle factors

\section{Introduction}

One of the important end-use performance parameters for fabrics and other textile products are fabric hands and fabric aesthetics. The Textile Institute [1] defines fabric hands as 'the quality of a fabric assessed by the reaction obtained from the sense of 
touch'. In a previous study, some principal factors governing fabric hands were proposed based on this definition. Howorth et al. [2] attempted to obtain the adjectives that affect the fabric hands using multiple factor analysis, and tentatively identified smoothness, stiffness, and thickness. Similarly, Kawabata [3] suggested five adjectives -smoothness, stiffness, crispness, fullness, and softness- for the worsted fabric of a men's suit. Following the findings of these studies, the Japanese industrial standards prescribed these adjectives as the principal factors that affect the fabric hands [4].

On the other hand, there have been some extensive theoretical and practical investigations that have aimed to define the fabric aesthetics. In one such study conducted by Brand [5], fabric aesthetics was examined as a relationship among at least six properties, namely style, body, cover, surface texture, drape, and resilience. Hoffman [6] suggested 36 effective adjectives for distinguishing fabrics of high and low aesthetics following a detailed evaluation. Nevertheless, fabric aesthetics have not yet been defined in a standard way. In our previous paper [7], fabric aesthetics was defined as 'the quality of a fabric assessed by the reaction obtained from the sense of sight', expanding upon the definition of fabric hands [1]. Then, as a first step in the systematic study of fabric aesthetics, we attempted to obtain the properties that affect the fabric aesthetics using factor analysis, and identified two principal factors tentatively labelled as 'lustre and depth sensation' and 'surface roughness sensation'.

The focus of the second step in the study of the fabric hands and fabric aesthetics is on the investigation of the objective trends in the performance evaluation of textiles because of inherent difficulties arising from the subjective evaluation, such as ambiguousness. The technique currently used for the subjective estimation of the fabric hands using physical properties has contributed to the establishment of the standards for quality control and the promotion of manufacturing efficiency on the development process of new textile products.

In the past, Kawabata et al. [3] developed a comprehensive measurement system for 
measuring the dynamic properties of woven fabrics (i.e. the tensile, shear, bending, compression, and friction properties), and proposed a characterization method as a means of estimating a fabric hands obtained by tactile perception.

On the other hand, the development of photometric equipment suitable for the quantitative evaluation of fabric aesthetics has been attempted by researchers [8-14]. In particular, goniophotometric measurements provide an effective means for the objective evaluation of lustre. Jeffries [8] constructed his own equipment for three-dimensional goniophotometric measurements, and investigated the degree of delustring for filament fabrics. Sawaji [9] also proposed his own equipment, which is able to arbitrarily set the angle of the sensor's optical axis as opposed to the perpendicular line of the sample holder, and defined an effective index for the evaluation of lustre. Li et al. [10] proposed equipment for three-dimensional goniocolorimetric measurements, and investigated how the surface geometric structure of woven fabrics affects the colorimetric measurements. For the sake of accuracy, these equipments are used to measure the mean value of the light reflection intensity of the fabric surface. However, considering that the fabric aesthetics is strongly affected by the distribution of the light reflection intensity, the microscopic goniophotometry can also prove to be an important tool, as previously mentioned in past papers [11-13].

Recently, Kim et al. [14] presented a novel method to analyze fabric images and estimated lustre. The method is based on the distribution of the light reflection intensity of the fabric under test and follows the goniophotometric principles.

Taking into account the previously mentioned technique, in this paper, we propose our own experimental equipment for microscopic goniophotometric measurements and a novel parameterization method. Then, we attempt to define an index based on the distribution of the light reflection intensity on woven fabrics and investigate its effectiveness in grading the principal factors (i.e. lustre and depth sensation and surface 
roughness sensation) governing fabric aesthetics, which have been identified in our previous study.

\section{Identification of principal factors governing fabric aesthetics [7]}

In our previous study, a number of textile experts and untrained consumers were asked to evaluate the aesthetics of worsted and spun silk woven fabrics with different structures (plain, twill, and satin). All the samples were dyed black as mentioned below. Twenty-six properties were classified into five categories: 'Lustre', 'Depth of dyeing', 'Roughness', 'Handling', and 'Preference'. It should be mentioned that nine Japanese experts from the textile industry and twenty untrained Japanese individuals evaluated fifteen paired samples with the above-mentioned properties based only on the sense of sight.

Thereafter, the evaluation scores were examined by means of factor analysis in order to identify the principal factors governing fabric aesthetics. According to the findings, two common factors -lustre and depth sensation and surface roughness sensation- were identified tentatively as the principal factors governing fabric aesthetics by both textile experts and untrained consumers. The factor scores of each sample are shown in Figure 1. The factor scores of lustre and depth sensation and surface roughness sensation were classified according to the difference of sample's materials or that of the sample's structure. In other words, the lustre and depth sensation were mainly affected by the material effects, while the surface roughness sensation was affected by the structural effects.

\section{Samples}

Six samples were woven especially for the needs of our experiments in three different weaves -plain, twill, and satin- using worsted and spun silk yarn. The yarn count for 
both the worsted and spun silk yarn was $16.7 \times 2$ tex. The weave density was 60 ends and 55 picks per $2.54 \mathrm{~cm}$ on the plain fabric, 90 ends and 70 picks per $2.54 \mathrm{~cm}$ on the twill fabric, 110 ends and 65 picks per $2.54 \mathrm{~cm}$ on the satin fabric.

These samples were dyed black using a reactive dye and a mordant dye so that the black colour was similar for all samples. The colour of the samples (i.e. $L^{*} a^{*} b^{*}$, as described by CIE [15]) was evaluated using five specimens per each sample by means of a colorimeter (Konica Minolta, CR-400), as shown in Figure 2. As the differences of the value, hue and chroma of the six woven fabrics were quite small, all samples were considered to be of the same colour.

\section{Experiment 1: Instrumentation for microscopic goniophotometry}

\section{Experimental}

Goniophotometric system, which is able to measure the distribution of the reflection intensity, was constructed experimentally, as shown in Figure 3 (hereinafter referred to as the 'experimental equipment'). The experimental equipment consisted of three movable parts (i.e. sample holder, incident light unit, and photometric unit). A rotation stage with a hollow part of $80 \mathrm{~mm}$ diameter was used as a sample holder for the measurement of light reflection on the surface of textiles. Specifically, each sample was placed between two supplemental plates, which were fixed on the sample holder.

The incident light source (Mejiro Precision Co., PHL-150) was connected using a fibre bundle to a focus lens and a diffusion board, in order to illuminate uniformly samples as shown in Figure 3(B). The illuminance at the surface of each sample was approximately $1000 \mathrm{~lx}$. The incident angle $(\alpha)$ was defined as the angle formed between the light source's optical axis and the direction perpendicular to the sample holder.

The photometric unit consisted of a neutral density (ND) filter, a focus lens, a charge-coupled device (CCD) camera (Hamamatsu Photonics, C3077-70) which is 
equipped with an array sensor and a personal computer, as shown in Figure 3(C). Images were acquired using a personal computer, which had a built-in image capture board (Library Co., cosmos), and quantized to an 8 bit grey level (256 gradations). The photometric angle $(\beta)$ was defined as the angle formed between the CCD camera's optical axis and the direction the perpendicular to the sample holder.

In order to be able to use the CCD camera as a luminance sensor, it is necessary to establish the relationship between image brightness (i.e. grey level of each pixel, hereinafter referred to as the 'grey level') and luminance when the settings of the optical system (e.g. limiting aperture and shutter speed) are fixed. Generally, this relationship is described by Equation (1), where $A_{F}$ is a constant determined by the settings of the optical system, and $\gamma$ is a constant determined by the characteristics of the photoelectrical sensor. Both $A_{F}$ and the $\gamma$ were estimated experimentally.

A grey-scale chart (Murakami Color Research Laboratory Co., GS-5) was illuminated uniformly by means of the light source found in the irradiation unit. The chart was graded eleven different levels. The luminance of each level was measured with a luminance meter (Topcon, BM-9) and the CCD camera. The grey-scale chart, the light source, the luminance meter, and the photometric unit were arranged as shown in Figure 4. The angular aperture of the luminance meter was $2^{\circ}$. The distance between the grey-scale chart and the luminance meter (or the photometric unit) was $260 \mathrm{~mm}$. The condition of exposure was set in eight cases (i.e. limiting aperture $\mathrm{f}=16,11,8,5.6,4$, $2.8,2,1.8$ and the shutter speed $=1 / 60 \mathrm{tps}$ ).

$V=A_{F} \cdot E^{\gamma} \cdots(1)$

where

$V$ denotes the grey level 
$E$ denotes the luminance

$A_{F}$ denotes a constant that is determined by the settings of the optical system $\gamma$ denotes a constant which is determined by the characteristics of the photoelectrical sensor

\section{Result and Discussion}

Figure 5 (a) show the relationship between the luminance $(E)$ and the grey level $(V)$. In spite of the increments of luminance, the gray level did not change lineally at the range of $V<20$ and $V>239$.

Hence a regression analysis using the data set of the value- $E$ and value- $V$ at the range of $20 \leqq V \leqq 239$ for the purpose of describing the relationship was conducted separately for each exposure. The constants $A_{F}$ and $\gamma$ were obtained as shown in Figure5 (b). Each equation was regressed statistically significant at the $p<0.01$ level, as shown in peason product-moment correlation coefficient (r). In other words, Equation (1) was used for the transformation of the grey level into luminance for each pixel.

\section{Experiment 2: Microscopic Photometry and GonioPhotometric Measurement Experimental}

The goniophotometric measurement is one of the effective means to assess fabric lustre [8-9]. It should be an efficient indicator for estimating the sensory aesthetics, because logically the property of photometric angle dependency involves the difference of features according to materials and structures of fabrics. However the human observer can only base his/her aesthetics judgment on perceptual static image such as a distribution of light reflection intensity at a certain observation condition. One of our interests on estimation of fabric aesthetics is either which means can be better predictor for assessing the fabric aesthetics. Therefore, the microscopic photometry and goniophotometric measurement were both conducted in the experiment. 
Five specimens per each sample were prepared in order to obtain the luminance distribution for the samples. The size of each specimens testing was $90 \times 90 \mathrm{~mm}$. Every specimen was placed at the sample holder with the warp yarn aligned with the sensor's optical axis.

Because the photometric angle $(\beta)$ was set arbitrarily, images were acquired at $-10^{\circ}$, $0^{\circ}, 10^{\circ}, 20^{\circ}, 30^{\circ}, 40^{\circ}, 50^{\circ}$, and $60^{\circ}$ with respect to the perpendicular direction on the fabric's surface. In contrast, the incident angle $(\alpha)$ was maintained stable at $30^{\circ}$ which was the same incident condition as that of sensory evaluation conducted in the previous study.

The limiting aperture of camera lens was either 2.8 or 2.0 , and shutter speed of CCD camera was 1/60 tps. The measurements were carried out in an environment of $24.8 \pm$ 0.4 and $40 \pm 5 \%$ R.H.

\section{Result and Discussion}

The size of the captured images was 640 pixels along the $\mathrm{x}$-axis, and 480 pixels along the y-axis (i.e. Video Graphics Array (VGA)), as shown in Figure 6(A). Nonetheless, the analysis of the captured images was carried out in an area of the central $101 \times 101$ pixels (angle of view: $\left.(3.1 \times 2.7)^{\circ}\right)$, which was selected in accordance with the area covered by the fovea centralis in the eye's retina [16]. It is estimated that the information perceived by the fovea centralis affects the subjective assessment of fabric aesthetics, because the cone cell at the fovea centralis is much more existing than any other part of retina (i.e. perception ability is much higher) (c.f. Figure 6(B)).

The grey levels of all pixels covering the analysis area were transformed into the luminance using Equation (1). The luminance distribution curves (hereinafter referred to as 'LDC') for the three worsted fabrics are shown in Figure 7. The $\mathrm{x}-\mathrm{y}$ plane and $\mathrm{z}$ axis correspond to the coordinate system of each fabric image and the luminance value at a specific position. 
There were many protrusions at the surface of the LDC. A shape of the protrusions was attributed to the state of each interlace between a warp and a weft yarn. In other word, the reflection at the floating yarn and surrounding interlaces decide the geometrical specification of the protrusion. And sequences of these protrusions were attributed to individual fabric structure, such as the parallel diagonal lines in LDC of twill weave (c.f. Figure $7(b))$.

\section{Experiment 3: Parameterization of Luminance Distribution}

\section{Experimental}

Assuming a distribution of light reflection intensity at the surface of fabric can governs perception of fabric aesthetics, obtaining the geometrical specification of the LDC by profiling these protrusions is one of the approach for prediction of fabric aesthetics. Hence the LDC was attempted to decompose into minimum components.

The minimum component was defined as a protrusion that comprises one luminance peak and its surrounding pixels that should have the lowest luminance in all eight-neighborhood directions (i.e. Moore-neighbourhood direction). Two image processing as 'neighborhood searching' and 'Moore-neighborhood tracing' were applied to the LDC in order to detect the area covered by the minimum components. Firstly, the local coordinates $\left(x_{\text {peak }}, y_{\text {peak }}\right)$ of pixels with the highest luminance value as compared with the pixels found in the eight-neighborhood directions were searched using a $3 \times 3$ pixel mask (c.f. Figure $6(\mathrm{C}))$. Secondly, the local coordinates $\left(x_{\text {valley }}, y_{\text {valley }}\right)$ of the pixel with the lowest luminance value in the eight-neighborhood directions around the pixel $\left(x_{p e a k}, y_{p e a k}\right)$ were searched for by means of 'Moore-neighborhood tracing'. As shown in Figure 8 , the local coordinate $(i, j)$ of the pixel where luminance was equal to $10 \mathrm{~cd} / \mathrm{m}^{2}$ was defined as the peak component (i.e. $L(i, j)>L(i-1, j-1) \wedge L(i, j)>L(i-1, j) \wedge L(i, j)>$ $L(i-1, j+1) \wedge L(i, j)>L(i, j-1) \wedge L(i, j)>L(i, j+1) \wedge L(i, j)>L(i+1, j-1) \wedge L(i, j)>L(i+1, j)$ 
$\wedge L(i, j)>L(i+1, j+1)$, where, $L$ denotes the luminance). Then the local coordinate $(\mathrm{i}+3, \mathrm{j})$ of the pixel where luminance was equal to $5 \mathrm{~cd} / \mathrm{m}^{2}$ was defined as the edge of right end of the minimum component. Similarly, the local coordinates $(i+2, j-2),(i, j-3),\left(j^{-} 3, j^{-3}\right)$, $(i-3, j),(i-2, j+2),(i, j+3),(i+3, j+3)$ were defined as the upper right, upper side, upper left, left side, lower left, lower side and lower right edge of the minimum component.

Subsequently, the area covered by each minimum component was estimated for all images acquired and the LDC was decomposed in minimum components.

Eight parameters were proposed for the parameterization of the LDC and their calculation was conducted according to the following definition (c.f. Figure 9).

\section{(1) Peak height of the minimum component $(H P)$}

$$
H P\left(c d / m^{2}\right)=\frac{\sum_{c o m p=1}^{N} L_{c o m p}\left(x_{\text {peak }}, y_{\text {peak }}\right)}{N} \ldots
$$

where

$L$ denotes the luminance of the pixel with local coordinates $(x, y)$

$\left(x_{p e a k}, y_{p e a k}\right)$ denotes the local coordinates of the pixel with the largest luminance with the minimum component

$N$ denotes the total numbers of minimum components

(2) Valley height of the minimum component $(H V)$

$$
H V\left(c d / m^{2}\right)=\frac{\sum_{c o m p=1}^{N} \sum_{\text {valley }=1}^{8} L_{\text {comp }}\left(x_{\text {valley }}, y_{\text {valley }}\right)}{8 \times N} \ldots
$$

where

$\left(x_{\text {valley }}, y_{\text {valley }}\right)$ denotes the local coordinates of all eight pixels found at the ends of the minimum component 
(3) Mean height of the minimum component (HM)

$H M\left(c d / m^{2}\right)=\frac{\sum_{c o m p=1}^{N} \sum_{p i x=1}^{n} L_{c o m p}\left(x_{p i x}, y_{p i x}\right)}{n \times N} \ldots$

where

$\left(x_{p i x}, y_{p i x}\right)$ denotes the local coordinates of all the pixels that compose the minimum component

$n$ denotes the total numbers of pixels composing the minimum component.

(4) Depth of the minimum component (DP)

$D P\left(c d / m^{2}\right)=H P-H V \cdots(5)$

(5) Width of the minimum component $(W)$

$W($ degree $)=\frac{\sum_{c o m p=1}^{N} w_{\text {comp }}}{N} \cdots(6)$

where

$w_{\text {comp }}=\frac{\sum_{d i r=1}^{4} \text { length }_{d i r} \times \text { pitch }}{4}$

length denotes the numbers of pixels found between two edges of the minimum component

pitch denotes a resolving power in the direction from the left to the right side or from the upper to the lower side (i.e. pitch $_{x}=\left(2.7 \times 10^{-2}\right){ }^{\circ}$ in the direction from left to right side, pitch $_{y}=\left(3.1 \times 10^{-2}\right)^{\mathrm{o}}$ in the direction from the upper to the lower side $)$

(6) Slope of the minimum component $(S)$ 
$S\left(\mathrm{~cd} / \mathrm{m}^{2} /\right.$ degree $)=\frac{D P}{W / 2} \cdots(7)$

\section{(7) Energy of the minimum component $(E)$}

The energy of the minimum component is defined as its cubic volume, as follows:

$E\left(\right.$ degree $\left.^{2} \cdot \mathrm{cd} / \mathrm{m}^{2}\right)=\sum_{\text {comp }=1}^{N} \sum_{p i x=1}^{n}\left\{L_{c o m p}\left(x_{\text {pix }}, y_{\text {pix }}\right) \times\right.$ pitch $_{x} \times$ pitch $\left._{y}\right\} \cdots$

\section{(8) Density of the minimum component (DENS)}

The density of the minimum component is defined as the ratio of the total numbers of minimum components and the size of the analysis area, as follows:

$$
\text { DENS }\left(\text { pieces } / \text { degree }^{2}\right)=\frac{N}{\text { Size }} \cdots(9)
$$

where

Size $\left(\right.$ degree $\left.^{2}\right)=101 \times$ pitch $_{x} \times 101 \times$ pitch $_{y}$

Size denotes the analysis area in angle of view (c.f. Figure 6(B))

\section{Result and Discussion}

In order to confirm the features of individual sample such as the material and structural effect were involved in the property of photometric angle dependency obtained by the goniophotometric measurement, the space reflectance curves on each parameter were shown in Figure 10 respectively. According to the results of space reflectance curves, interpretation of the physical meanings of eight parameters was discussed below.

The space reflectance curve of $H P$ was classified according to the difference of the sample's materials (c.f. Figure 10(a)). There were significant differences between the 
values of $H P$ at an angle $(\beta)$ of $0^{\circ}$ in all the pairs of worsted and spun silk fabric. (ANOVA, Fisher's LSD, $\mathrm{p}<0.01$ level). In addition, the peak was observed at the angle ( $\beta$ ) of $30^{\circ}$ which was specular condition on the satin of spun silk fabric. There were also significant differences among the values of $H P$ at an angle $(\beta)$ of $30^{\circ}$ in the pair of satin and fabrics with other weave patterns. (ANOVA, Fisher's LSD, $\mathrm{p}<0.01$ level). Similarly, $H V, H M, D P, S$ and $E$ of the minimum component exhibited the same tendency as $H P$ (c.f. Figure 10(b-f)). Six parameters (i.e., $H P, H V, H M, D P$, $S$ and $E$ ) out of eight were highly correlated each other $(p<0.01$ level). That is to say, either six were the parameters concerned to the intensity of local reflection.

In contrast, the space reflectance curve of $W$ and DENS exhibited totally different patterns from that of $H P$ (c.f. Figure $10(\mathrm{~g})$ and (h)). Namely, no notable peaks were observed in any samples at any photometric angle. In the case of plain weave, the $W$ of the minimum component was the lowest among other woven fabrics, because the floating yarn appeared periodically and shortly on the fabric surface. That is to say, either six parameters physically mean local light reflection intensity. Similarly in the case of plain weave, the $D E N S$ of the minimum component was relatively high, because of the significantly larger numbers of floating yarns appearing on the surface of the plain weave. That is to say, DENS is a parameter concerned to the number of floating yarn at the physical aspect.

According to the result of space reflectance curve, it was confirmed that the material effect of each sample was obtained by the goniophotometric measurement of the $H P, H V$, $H M, D P, S$ and $E$. On the other hand, the structural effect was obtained by that of the $W$ and DENS. 


\section{Experiment 4: Prediction of Fabric Aesthetics}

\section{Experimental}

As we mentioned above, the main purpose of this study is to propose a predictable equation for two principal factors (i.e., 'lustre and depth sensation' and 'surface roughness sensation'). At this point, the efficiency of the novel parameters as independent variables obtained by microscopic photometry would be investigated in comparison with the conventional parameter obtained by the goniophotometric measurement.

As we found in Experimental 3, either six parameters (i.e., $H P, H V, H M, D P, S$ and $E$ ) out of eight represented a similar physical meaning concerned to the local intensity of light reflection. That is to say, the eight parameters involved redundancy on the decision of the independent variable. And, either eight parameters were induced directly according to the specification of woven fabrics such as the material and the structural effect. As more direct predictors to reduce the redundancy of eight parameters and to correlate with principal factors, two parameters - BRIGHTNESS, FINENESS - were newly produced by combination of eight parameters.

The BRIGHTNESS which was produced by products of $E$ and $D E N S$ would be assumed to become a predictor for 'lustre and depth sensation'. The $E$ and DENS were the indicators for the reflection energy and the numbers of minimum component respectively. In a previous study, we reported that the ratings of lustre and depth sensation were highly loaded by ratings of some attributes of fabric aesthetics representing brightness (i.e., deep, dark, shiny, rich highlights and glittery) [7]. From the findings, it was expected that the larger the $E$ and $D E N S$, the higher the ratings of sensory brightness.

Similarly, the FINENESS which was produced by products of $W$ and DENS would be assumed to become a predictor for 'surface roughness sensation'. The $W$ and $D E N S$ were 
the indicators for the length of floating yarn and the numbers of minimum component respectively. In a previous study, the ratings of surface roughness sensation were highly loaded by the ratings of some attributes of fabric aesthetics representing sensory fineness (i.e., delicate, fine, sleek) [7]. From the findings, it was expected that the larger the $W$ and $D E N S$, the higher the rating of sensory fineness.

The correlation matrix among the ratings of principle factors and scores of two parameters are shown in table 1 . The ratings of lustre and depth sensation were correlated significantly with the scores of BRIGHTNESS at the $\mathrm{p}<0.01$ level, but no significant correlation coefficient was observed with scores of FINENESS. In contrast, the ratings of surface roughness sensation were correlated significantly with the scores of FINENESS at the $\mathrm{p}<0.05$ level, but no significant correlation coefficient was observed with scores of BRIGHTNESS. Thus these two parameters were expected to be better predictors for two principle factors.

A linear multiple regression model (c.f. Equation (10)) was applied to evaluate the relationship between dependent and independent variables. The dependent variable was the factor scores of 'lustre and depth sensation' and that of 'surface roughness sensation' (c.f. Figure 1). In contrast, the independent variables were above-mentioned two parameters (i.e., BRIGHTNESS and FINENESS). The variable selection was conducted with 'stepwise method' which is 'forward selection method' with possibility of excluding the once selected variable. Consequently, the standard regression coefficients were calculated for the two principal factors respectively.

$\mathrm{v}^{\text {dep }}=\beta_{0}+\beta_{1} \cdot v_{1}^{\text {ind }}+\beta_{2} \cdot v_{2}^{\text {ind }}+\cdots+\beta_{p-1} \cdot v_{p-1}^{\text {ind }}+\beta_{p} \cdot v_{p}^{\text {ind }}+\varepsilon \cdots(10)$

where

$\mathrm{v}^{\text {dep }}$ denotes a dependent variable

$\mathrm{v}^{\text {ind }}$ denotes an independent variable 
$\beta$ denotes the standard regression coefficient

$\varepsilon$ denotes an error term

$p$ denotes the numbers of independent variables

In contrast, the data set of $\mathrm{LDC}$ for each sample was analyzed according to a conventional method proposed by Sawaji for the case of goniophotometric measurement [9]. The index of the diffused gloss is calculated as the ratio of the 'total reflectance at different angles' and the 'specular reflectance' measured by means of goniophotometry, as shown by the following equation:

$\operatorname{DIFF}($ a.u. $)=\frac{1}{A v e_{\text {spec }}} \sum_{\text {condition }-0}^{7}$ Ave $e_{\text {ang }}($ condition $\cdot \Delta \theta) \cdots(11)$

where

Ave denotes the average luminance of each pixel found within the portion of the LDC to be analyzed

ang denotes the photometric angle

Spec denotes specific conditions under which the photometric angle is the same as the incidence angle (i.e. $\alpha=30^{\circ}$ in this study)

Following a similar approach as the above-mentioned regression analysis was employed to describe mathematically the relationship between the 'DIFF and the factor scores of two principal factors. Specifically, the regression model described by Equation (12) was employed. In this case, the ordinary least square method was applied to DIFF, which was considered as an independent variable, and the factor scores of lustre and depth sensation and surface roughness sensation as dependent variables.

Consequently, the standard regression coefficients were obtained for two principal factors. 
$\mathrm{v}^{d e p}=\beta_{0}+\beta_{1} \cdot v_{1}^{\text {ind }} \ldots$

\section{Result and Discussion}

The regression model for the prediction of principal factors governing fabric aesthetics (i.e. lustre and depth sensation and surface roughness sensation) was defined, as shown by Equations (13) and (14) (hereinafter referred to as the 'proposed method'). In addition, two further regression models were obtained from the above-mentioned simple regression analysis which were used to evaluate the effectiveness of the proposed method, as shown by Equations (15) and (16) (hereinafter referred to as the 'conventional method').

According to Equation (13), the factor scores for lustre and depth sensation and two additional parameters (i.e. BRIGHTNESS and FINENESS) correlated positively with each other. We believe that the larger the $D E N S$ and the $E$ of the minimum component is, the stronger the lustre sensation is. Similarly, the larger the DENS and the $W$ of the minimum component is, the larger the number of cone cells activated in the retina is. Consequently, these two parameters were regarded equivalent to lustre and depth sensation.

In contrast, according to Equation (14), the factor scores for surface roughness sensation and one particular parameter (i.e. FINENESS) correlated positively with each other. As we mentioned earlier, the shape of minimum components of the LDC denote the property of light reflection on floating yarn. Hence, we believe that the longer the sequence of floating yarn is, the stronger the smoothness sensation is. Similarly, the larger the DENS of the minimum component is, the stronger the smoothness sensation is. Consequently, this parameter was regarded equivalent to surface roughness sensation. 


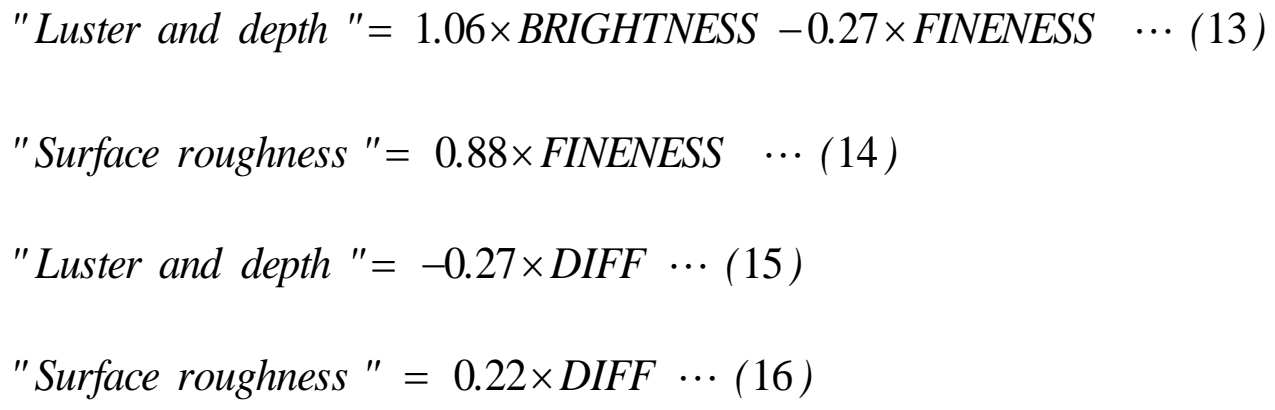

The relationships between experimental scores of lustre and depth sensation and scores predicted by Equations (13) and (15) are shown in Figure 11. Similarly, the relationships between experimental scores of surface roughness sensation and scores predicted by Equation (14) and (15) are shown in Figure 12. Using either conventional method or the proposed method, the correlation coefficient between experimental scores and predicted scores for lustre and depth sensation was relatively high (i.e. $R^{2}=0.98$ according to the proposed method and $\mathrm{R}^{2}=0.54$ according to the conventional method). In particular, not only the gradations that were influenced by material effects but also the gradations that were influenced by structural effects were accurately predicted by the proposed method.

In contrast, the experimental scores for surface roughness sensation were predicted with superior accuracy by the proposed method (i.e. $\left.\mathrm{R}^{2}=0.77\right)$ than by the conventional method (i.e. $\mathrm{R}^{2}=0.32$ ). However the type II error rate (B-rate) for the regression analysis of "surface roughness" was 0.38 in proposed method and 0.83 in conventional method. In order to remove the effect of type II error, the sufficient B-rate required lower than 0.2 in general. Hence a null-hypothesis could not reject completely. Because it was induced by a lack of the numbers of sample, the improvement of reliability on the result of statistical test with sufficient number of samples is necessary as a future consideration. 


\section{Summary}

In this paper, we proposed our own experimental equipment that was developed based on microscopic goniophotometric principles as well as a parameterization method. Subsequently, we defined an index for quantitatively estimating the fabric aesthetics based on the LDC of woven fabrics and eight parameters of its minimum components, which allow us to evaluate principal factors governing fabric aesthetics, such as lustre and depth sensation and surface roughness sensation, identified in our previous study. The following conclusions were obtained.

(1) The space reflectance curves for the peak height $(H P)$, valley height $(H V)$, mean height $(H M)$, depth $(D P)$, slope $(S)$, and energy $(E)$ were classified according to difference of materials. Hence, the influence of material effects is stronger than that of the structural effect on these six parameters. In contrast, the space reflectance curve for $\operatorname{width}(W)$ and density $(D E N S)$ were classified according to the weave pattern, because these parameters were influenced by the shape of the minimum components that defines the property of light reflection on floating yarn. In other words, the influence of structural effects is stronger than that of material effects on these two parameters.

(2) Based on the results of regression analysis, equations were developed for the prediction of two principal factors governing fabric aesthetics. Specifically, the BRIGHTNESS (i.e., product of DENS and E) and the FINENESS (i.e., product of DENS and $W$ ) were estimated to be equivalent to lustre and depth sensation. Similarly, the product of FINENESS can be regarded to be equivalent to the surface roughness sensation.

(3) Using the regression equation, the relationship between experimental scores and predicted scores was investigated. Specifically, the experimental scores for lustre and 
depth sensation were accurately predicted by both the conventional and the proposed method. In particular, not only the gradations that were influenced by material effects but also the gradations that were influenced by structural effects were accurately predicted by the proposed method. In contrast, the experimental scores for surface roughness sensation were predicted with more reasonable predictors by the proposed method than by the conventional method.

In all, these results suggest that the proposed method using principles of microscopic photometry and its characterization is a more viable approach than the methods based on conventional macroscopic goniophotometry for the quantitative evaluation of principal factors governing fabric aesthetics.

\section{Acknowledgments}

We wish to thank Mr Kentarou Nasu, graduate student at Shinshu University, for their assistance.

\section{Funding}

This study was supported by A Research for Promoting Technological Seeds Program (06-026) by Japan Science and Technology Agency.

\section{References}

1. Denton M. J., and Daniels, P. N., "Textile Terms and Definitions", 11th edn, The Textile Institute, London, 2002, p. 163.

2. Howorth W.S., The Handle of Suiting, Lingerie, and Dress Fabrics, J. Text. Inst., 49, T540 (1956)

3. Kawabata S., "The Standardization and Analysis of Hand Evaluation", 2nd edn,, 
HESC. Textile Mach. Sci. Jpn., Osaka, (1980)

4. Japanese Industrial Standards (JIS) L 0207, "Glossary of terms used in textile industry (Dyeing and finishing) (2005)

5. Brand R. H., Measurement of aesthetics analysis of aesthetic components, Textile Res.

J. $34,791-804(1964)$

6. Hoffman R. M., Measuring the Aesthetic Appeal of Textiles, Textile Res. J., 56, 428$434(1965)$

7. Kanai H., Morishima M., Nasu K., Nishimatsu T., Shibata K., Matsuoka T., Identification of Principal Factors of Fabric Aesthetics by the Evaluation of Experts on Textile and Untrained Consumers, Textile Res. J., 2011, DOI: 10.1177 / 00405175113999 60

8. Jeffries, R., Measurement of the Extent of Delustring of Filament Fabrics, J. Text. Inst., 46, 391-399 (1955)

9. Sawaji, M., A Device for Grading the Gloss of Textile Fabrics, Jpn. J. of Applied Physics, 29, 698-704 (1960)

10. Lee, W., Sato, M., Effect of the Surface Geometry of the Fabrics on Reflection of the Light, J. of the Color Sci. Assoc. of Jpn, 24, 140-145 (2000)

11. Sawaji, M., A Study on the Quality of Luster Micro-Structural Reflection Characterisitics, Jpn. J. of Applied Physics, 29, 789-794, (1960)

12. Mehdi, H., Dariush, S. and Mohammad, S., A New Method for Measuring Luster Index Based on Image Processing, Textile Res. J., September, 4, 2009, DOI: 10.1177 / 0040517509343814

13. Takeda, Y., Sakaguchi, Y., Tanaka, H., Efficient Image-based Anisotropic Reflectance Rendering of Silk-like Woven Fabrics, The J. of the Soc. For Art and Sci., 7, $132-144(2008)$

14. Shin K. I., Kim S. H., Kim J. J., Image Analysis of the Luster of Fabrics with 
Modified Cross-section Fibers, Fibers and Polymers, 6, 82-88 (2005)

15. ISO 11664-4 (CIE S 014-4/E) “Colorimetry - Part 4: CIE 1976 L*a*b* Colour space”, (2008)

16. Wiliam K. P., Digital Image Processing 2nd edn., A Wiley-Interscience publication, New York, 1991, p. 26. 
$\begin{array}{lll}\odot-\text { Plain (silk) } & \square \text { Twill (silk) } & \diamond \text { Satin (silk) } \\ -\odot-\text { Plain (worsted) } & -\bullet-\text { Twill (worsted) } & \diamond-\text { Satin (worsted) }\end{array}$

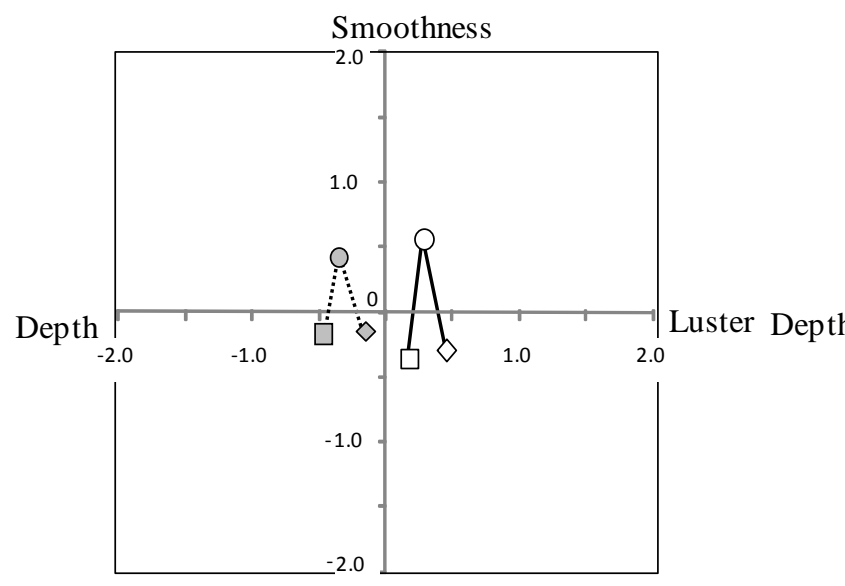

Roughness

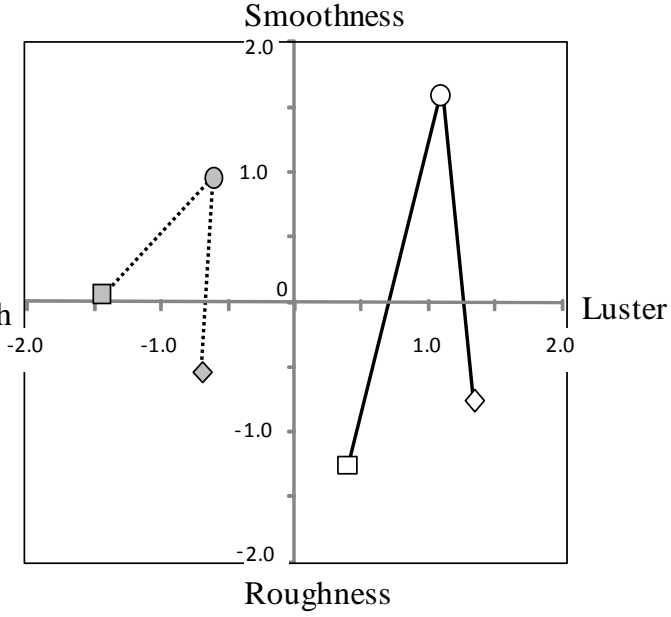

(b) Textile experts $(n=9)$

(a) Untrained consumers $(\mathrm{n}=20)$

Figure 1 Factor scores for lustre and depth sensation and surface roughness sensation [7] 


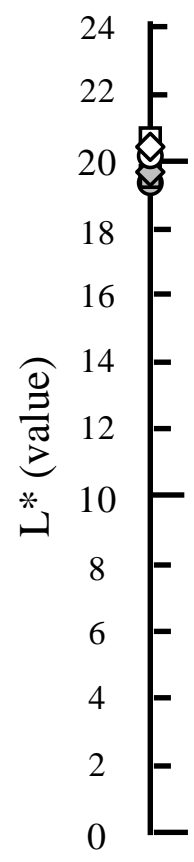

(a) $\mathrm{L}^{*}$ (value)

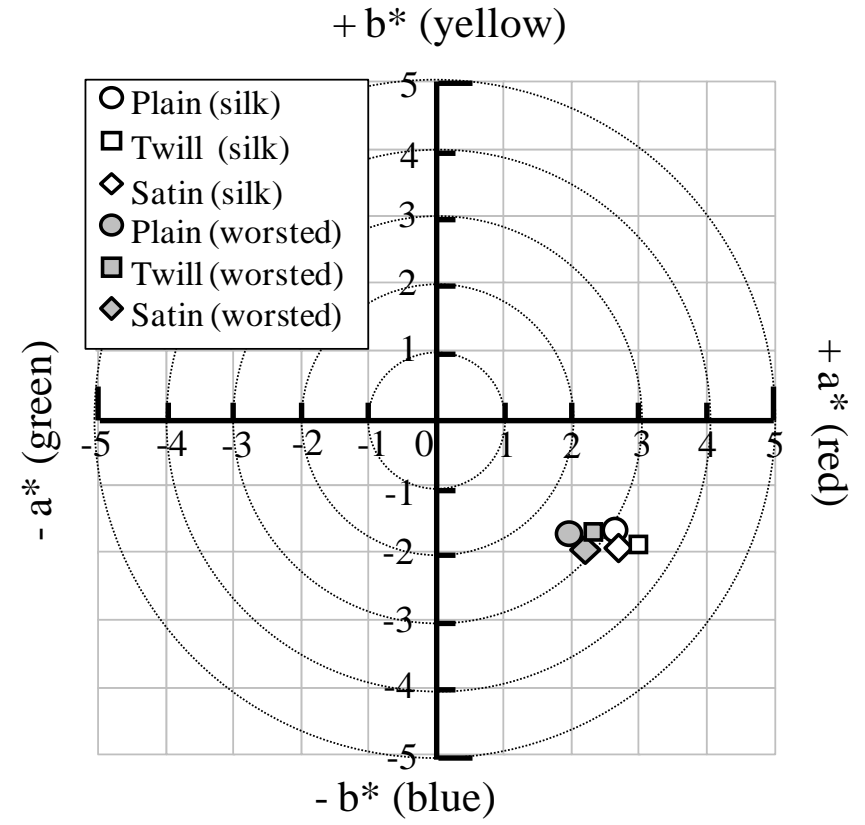

(b) Colour difference in $a^{*}-b^{*}$ space

Figure 2 Results of colorimetry performed on textile samples [7] 


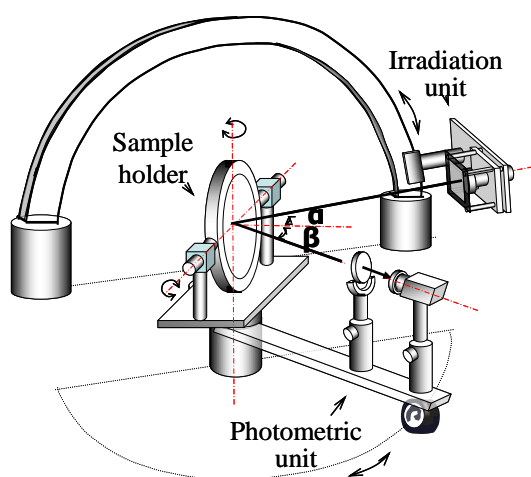

(a) Experimental equipment for microscopic photometry

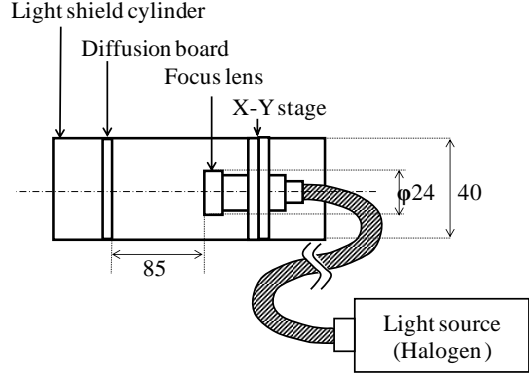

(b) Incident light unit (unit of measurement: $\mathrm{mm}$ )

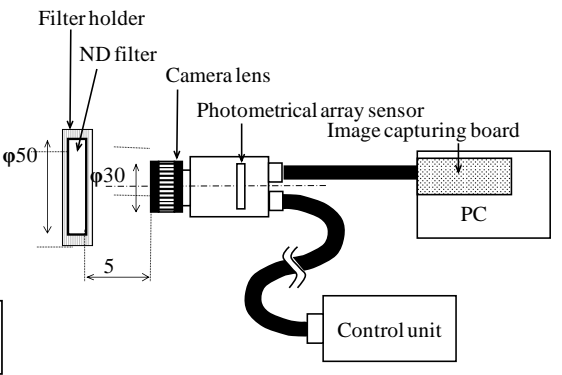

(c) Photometric unit (unit of measurement: $\mathrm{mm}$ )

Figure 3 Schematic diagrams of experimental equipment 


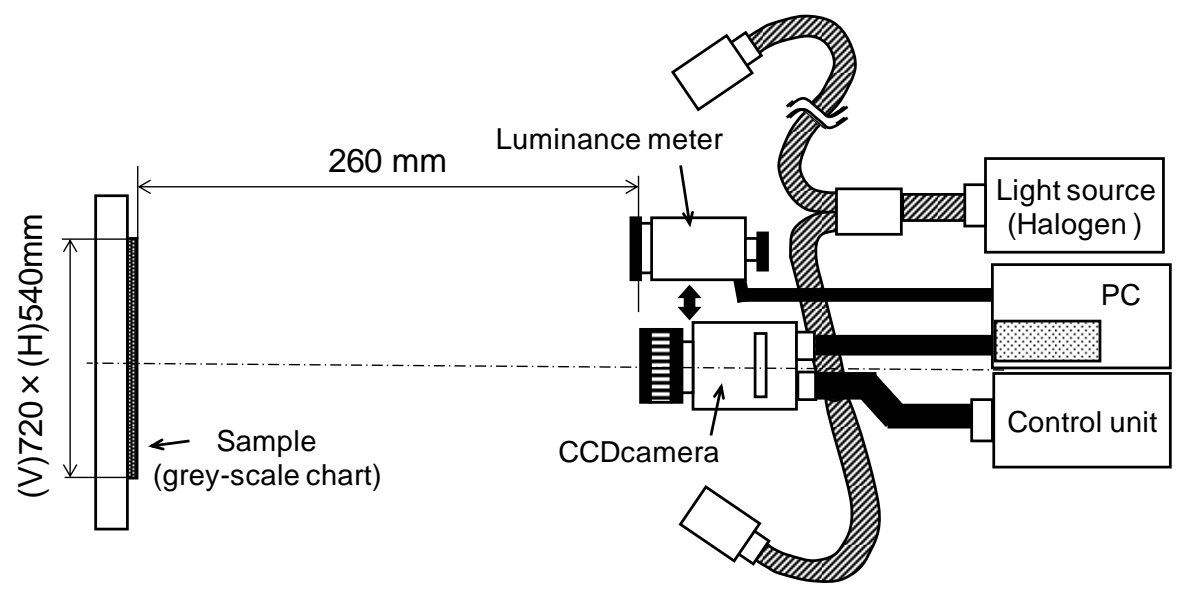

Figure 4 Experimental layout used for calibration of the grey levels against luminance values 


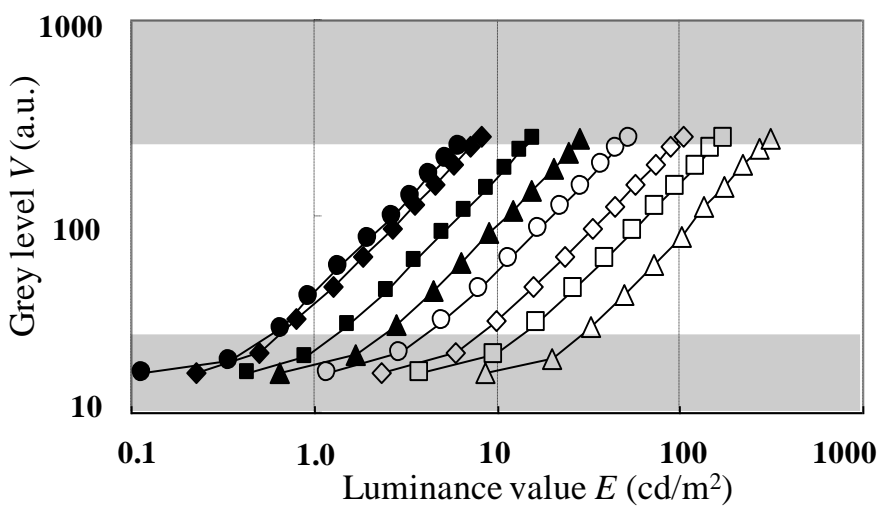

(a) Relationship between luminance and grey level

\begin{tabular}{ccccc}
\hline \hline & $F$ & $A_{F}$ & $\gamma$ & $\boldsymbol{r}$ \\
\hline- & 1.8 & 40.48 & 0.95 & 1.00 \\
\hdashline & 2.0 & 36.69 & 0.91 & 0.99 \\
\hdashline & 2.8 & 18.23 & 0.94 & 0.99 \\
\hdashline & 4.0 & 10.47 & 0.94 & 1.00 \\
- & 5.6 & 6.95 & 0.90 & 0.98 \\
\hdashline & 8.0 & 3.82 & 0.90 & 0.99 \\
$\square-$ & 11.0 & 2.07 & 0.93 & 0.99 \\
$-\triangle$ & 16.0 & 0.79 & 1.01 & 0.99 \\
\hline
\end{tabular}

(b) Values of constants $A_{F}$ and $\gamma$

Figure 5 Results of transformation of the grey level into luminance for each pixel $\mathrm{r}^{2}$ denotes the coefficient of the linear relation between the luminance value and grey level 
640 pixels

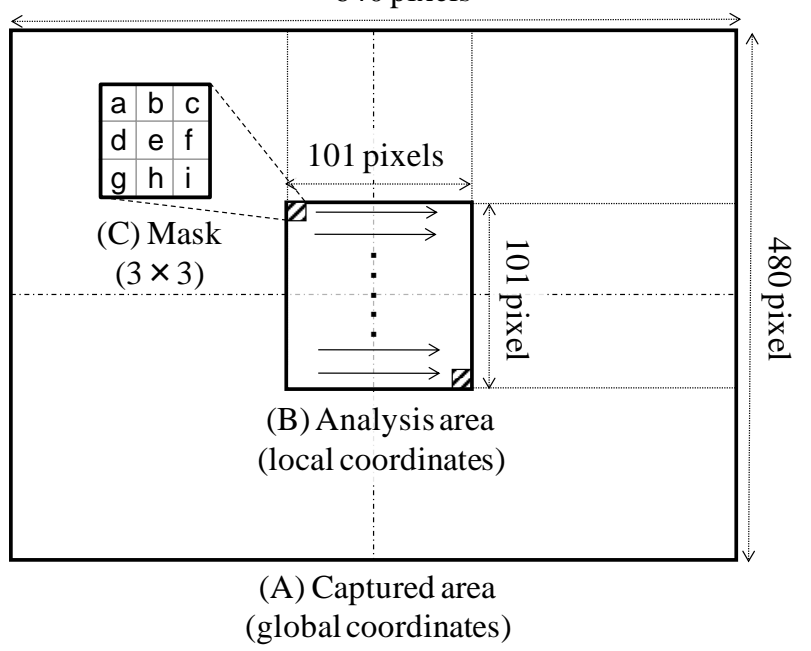

Figure 6 Extraction of analysis area from captured image 


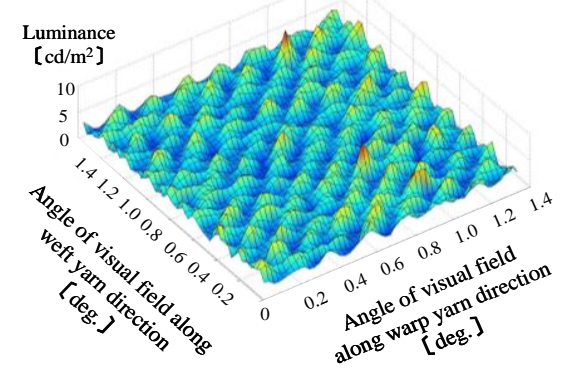

(a) Plain (worsted)

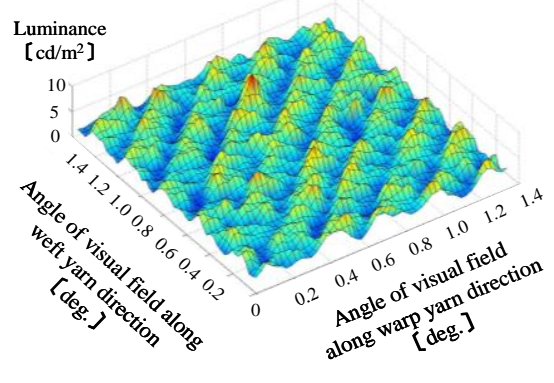

(b) Twill (worsted)

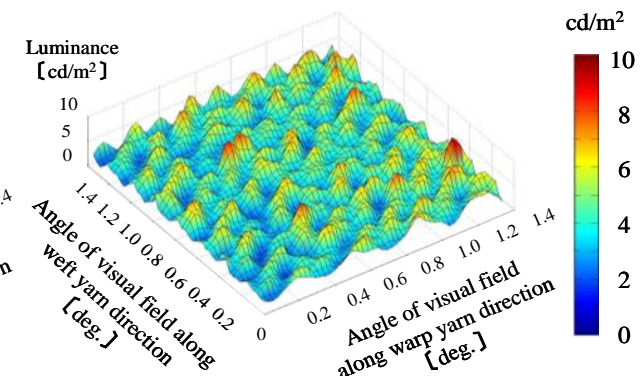

(c) Satin (worsted)

Figure 7 Luminance distribution curves (LDC) 


\begin{tabular}{|c|c|c|c|c|c|c|c|c|c|c|c|c|c|c|c|}
\hline & $x_{1}$ & 2 & . & $i-4$ & $i-3$ & $i-2$ & $i-1$ & $l$ & $l+$ & $i+2$ & $l+$ & $l+4$ & $\cdots$ & $x_{99}$ & $x_{100}$ \\
\hline \multirow{3}{*}{$\begin{array}{l}y_{1} \\
y_{2}\end{array}$} & 9 & 8 & \multirow{2}{*}{$\begin{array}{l}\ldots \\
\ldots\end{array}$} & 8 & 8 & 7 & 8 & 3 & 3 & 3 & 4 & 6 & \multirow{2}{*}{$\begin{array}{l}\ldots \\
\ldots\end{array}$} & \multicolumn{2}{|r|}{8} \\
\hline & 4 & 7 & & 5 & 5 & 6 & 5 & 4 & 3 & 2 & 3 & 5 & & 9 & 8 \\
\hline & $\vdots$ & $\vdots$ & : & $\vdots$ & $\vdots$ & $\vdots$ & $\vdots$ & $\vdots$ & $\vdots$ & $\vdots$ & $\vdots$ & $\vdots$ & & $\vdots$ & $\vdots$ \\
\hline$j-4$ & 8 & 7 & \multirow{2}{*}{$\begin{array}{l}\cdots \\
\cdots\end{array}$} & 6 & 7 & 6 & 6 & 7 & 5 & 6 & 6 & 7 & \multirow{2}{*}{$\begin{array}{l}\cdots \\
\ldots\end{array}$} & 6 & 7 \\
\hline \multirow{2}{*}{$\begin{array}{l}j-3 \\
j-2\end{array}$} & 5 & 4 & & 4 & 4 & 5 & 4 & 33 & 4 & 5 & 6 & 7 & & 6 & 7 \\
\hline & 6 & 5 & $\ldots$ & 5 & 5 & 5 & 6 & 6 & 7 & 5 & 5 & 6 & $\ldots$ & 6 & 5 \\
\hline$j-1$ & 7 & 6 & $\ldots$ & 6 & 6 & 5 & 9 & 8 & 8 & 7 & 6 & 7 & \multirow{2}{*}{$\begin{array}{l}\ldots \\
\ldots\end{array}$} & 7 & 6 \\
\hline \multirow{2}{*}{$\begin{array}{c}J \\
j+1\end{array}$} & 7 & 4 & . & 5 & 4 & 5 & 8 & 10 & 9 & 7 & 5 & 6 & & 4 & 2 \\
\hline & 6 & 5 & $\cdots$ & 5 & 5 & 6 & 9 & 9 & 7 & 6 & 5 & 4 & $\ldots$ & 2 & 3 \\
\hline$j+2$ & 5 & 4 & $\ldots$ & 4 & 4 & 7 & 8 & 5 & 3 & 6 & 4 & 5 & $\ldots$ & 3 & 4 \\
\hline \multirow{3}{*}{$\begin{array}{l}j+3 \\
j+4\end{array}$} & 7 & 8 & $\ldots$ & 8 & 8 & 6 & 7 & 2 & 2 & 2 & 5 & 5 & & 2 & 5 \\
\hline & 9 & 8 & $\ldots$ & 8 & 8 & 7 & 8 & 3 & 4 & 3 & 8 & 6 & & 3 & 3 \\
\hline & $\vdots$ & $\vdots$ & $\vdots$ & $\vdots$ & $\vdots$ & $\vdots$ & $\vdots$ & $\vdots$ & $\vdots$ & $\vdots$ & $\vdots$ & $\vdots$ & \multirow{3}{*}{$\begin{array}{l}\ldots \\
\ldots \\
\end{array}$} & $\bar{\vdots}$ & $\vdots$ \\
\hline \multirow{2}{*}{$y_{99}$} & 4 & 7 & $\ldots$ & 6 & 5 & 7 & 5 & 4 & 3 & 3 & 3 & 5 & & 9 & 8 \\
\hline & 9 & 8 & $\ldots$ & 7 & 9 & 7 & 8 & 5 & 4 & 3 & 3 & 6 & & 8 & 8 \\
\hline
\end{tabular}

\section{Area of minimum component of LDC}

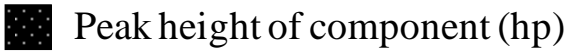

Valley height of component (hv)

Figure 8 Detection of minimum components of LDC 


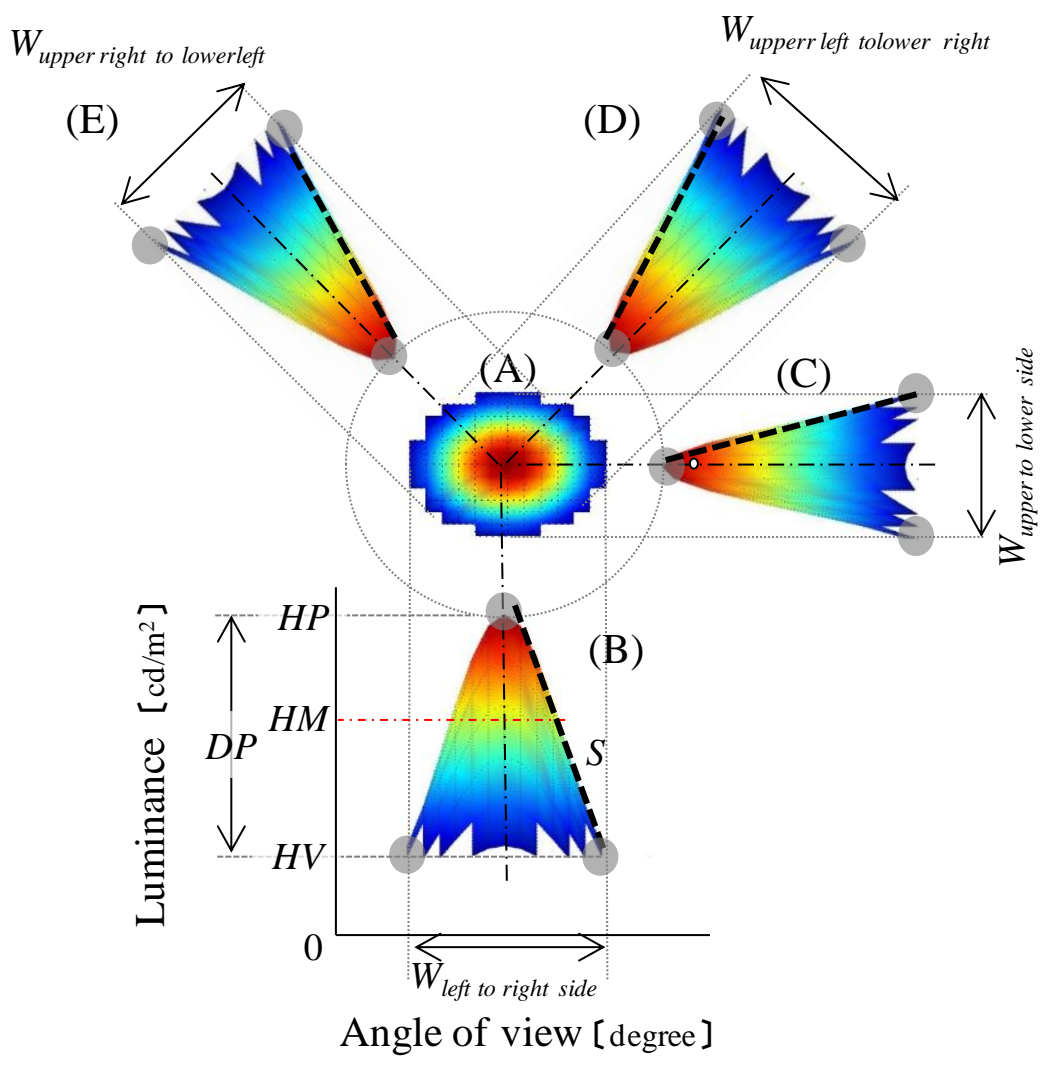

Figure 9 Minimum component and its parameterization

(A) Top view ( $x^{-} y$ plane), (B) Front view ( $x^{-} z$ plane), (C) Side view (y-z plane), (D) Side view (Bias-45 $45^{\circ}$ plane), (E) Side view (Bias- $135^{\circ}$ plane)

$H P$. Peak height, $H V$ : Valley height, $H M$ ः Mean height, $D P$. Depth, $W_{\text {dir }}$ Width (direction: 'left to right side', 'upper right to lower left', 'upper to lower side', 'upper left to lower right'), $S$. Slope. 


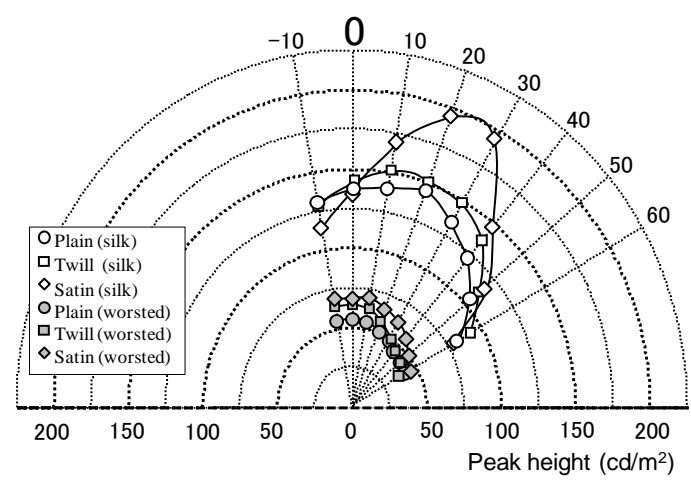

(a) Peak height of minimum component $(H P)$

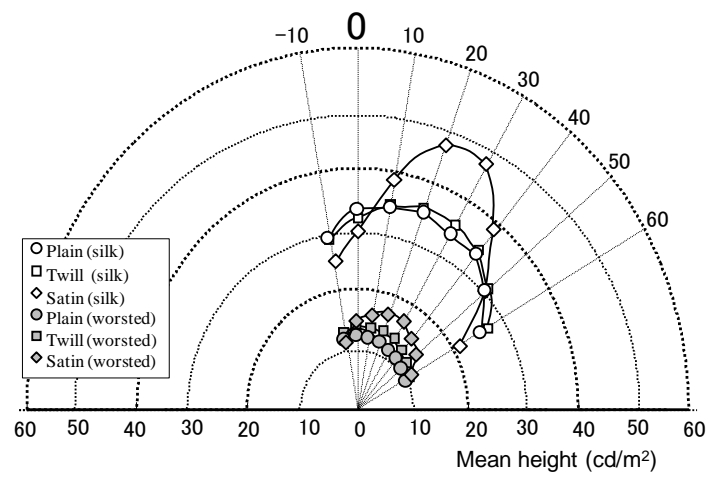

(c) Mean height of component (HM)

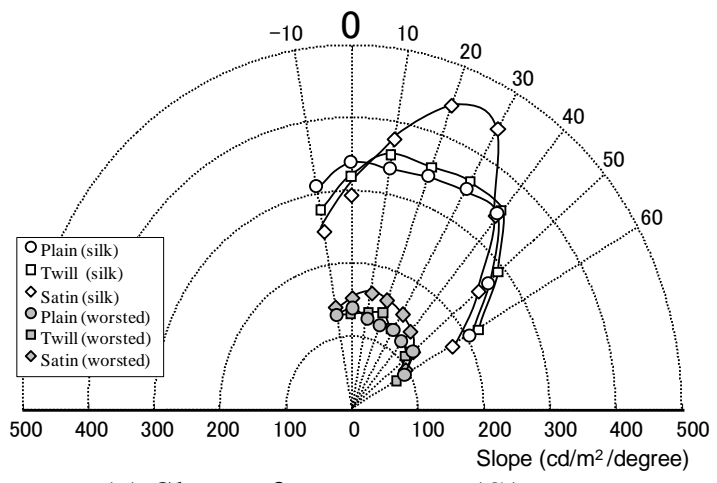

(e) Slope of component $(S)$

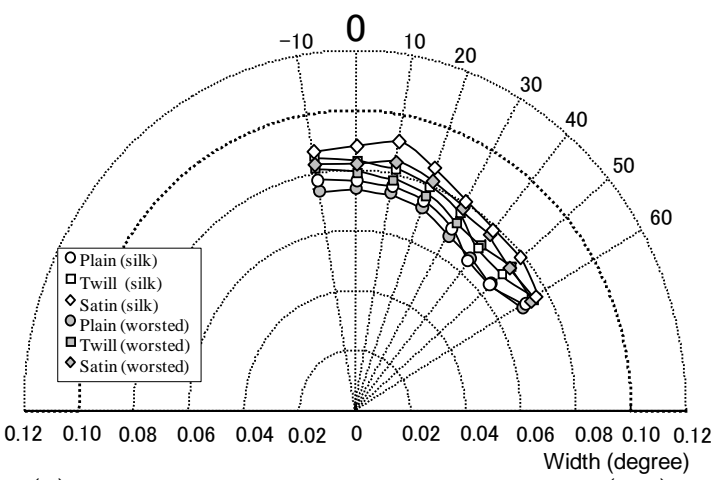

(g) Width of minimum component $(W)$

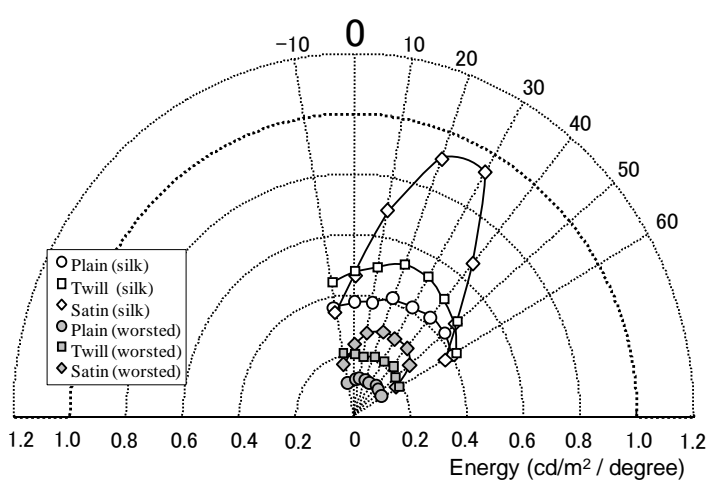

(b) Valley height of minimum component $(H V)$

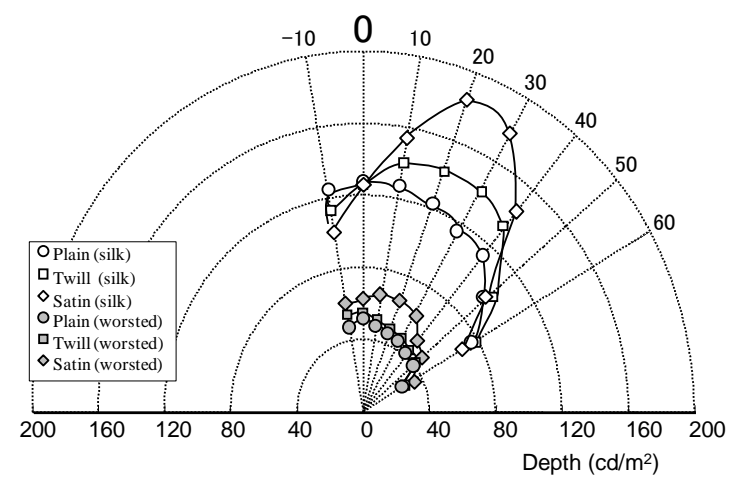

(d) Depth of component $(D P)$

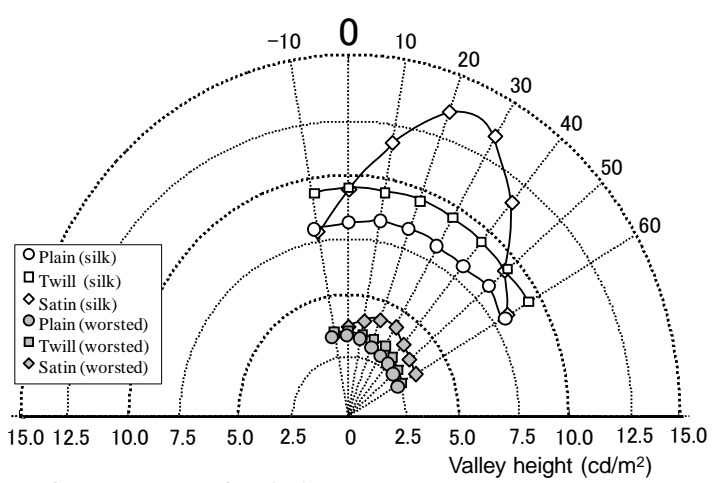

(f) Energy of minimum component $(E)$

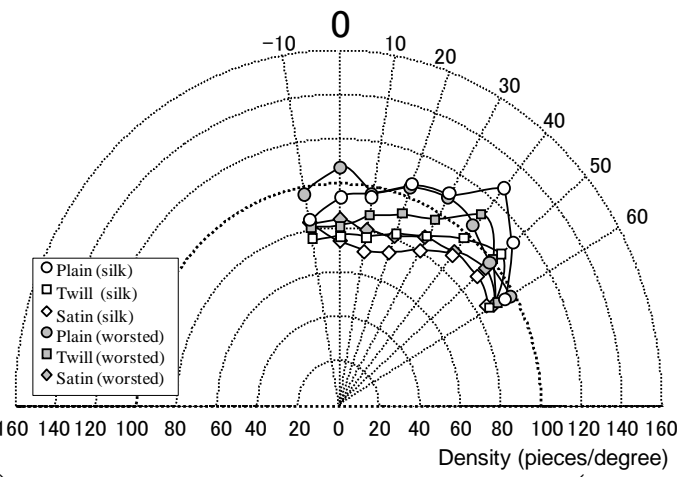

(h) Density of minimum component (DENS)

Figure 10 Space reflectance curves used for characterization of LDC 
Table 1 Matrix of correlation coefficient

\begin{tabular}{|c|c|c|c|c|c|}
\hline & & \multicolumn{2}{|c|}{ Principle factors } & \multicolumn{2}{|c|}{ Parameters } \\
\hline & & $\begin{array}{l}\text { Lustre and } \\
\text { depth }\end{array}$ & $\begin{array}{c}\text { Surface } \\
\text { roughness }\end{array}$ & BRIGHTNESS & FINENESS \\
\hline \multirow{2}{*}{$\begin{array}{l}\text { Correlation } \\
\text { coefficient }\end{array}$} & Lustre and depth & - & -0.115 & 0.956 & -0.123 \\
\hline & Surface roughness & -0.115 & - & -0.301 & 0.877 \\
\hline \multirow{2}{*}{$P$ - value } & Lustre and depth & - & 0.829 & 0.003 & 0.816 \\
\hline & Surface roughness & 0.829 & - & 0.562 & 0.022 \\
\hline
\end{tabular}




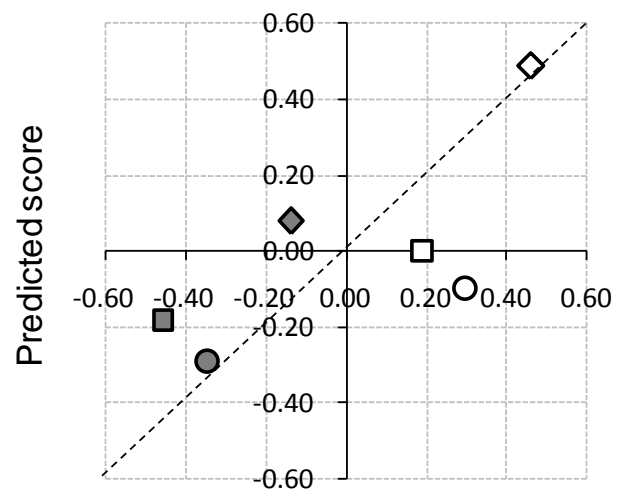

Experimental score

(a) Conventional method $\left(\mathrm{R}^{2}=0.54\right)$

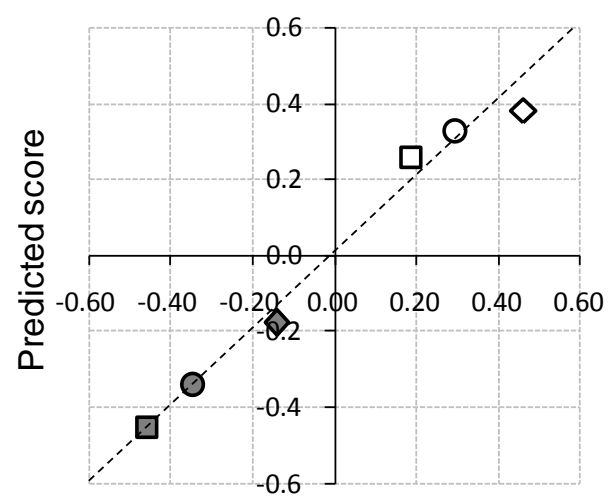

Experimental score

(b) Proposed method $\left(\mathrm{R}^{2}=0.98\right)$

Figure 11 Prediction of scores for lustre and depth sensation

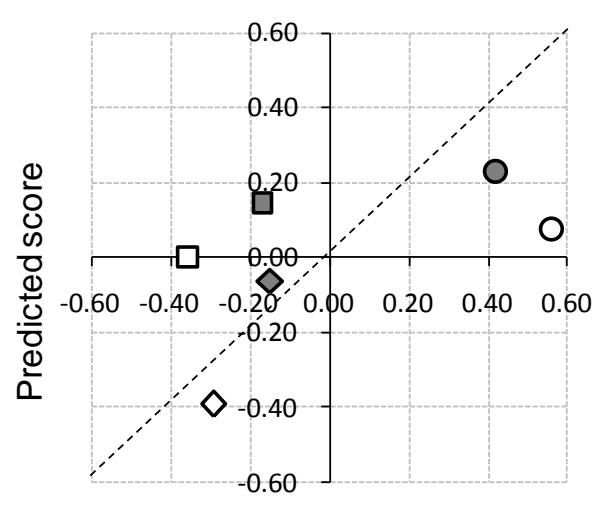

Experimental score

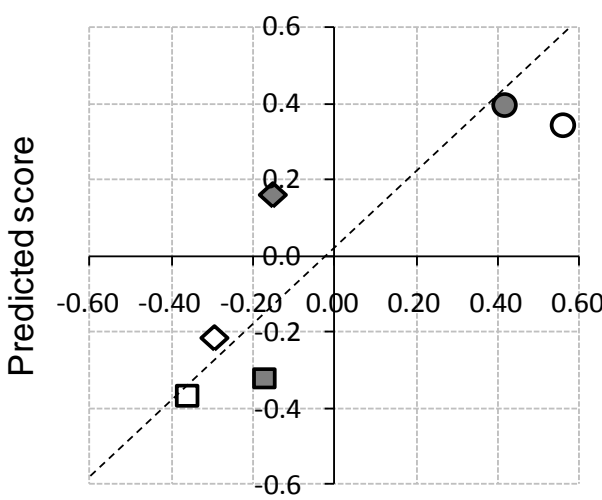

Experimental score

(b) Proposed method $\left(\mathrm{R}^{2}=0.77\right)$

(a) Conventional method $\quad\left(\mathrm{R}^{2}=0.32\right)$

Figure 12 Prediction of scores for surface roughness sensation 